\title{
Chemoprevention of Oesophageal Squamous-Cell Carcinoma and Adenocarcinoma: A Multicentre Retrospective Cohort Study
}

\author{
Junya Arai ${ }^{a}$ Ryota Niikura ${ }^{a}$ b Yoku Hayakawa ${ }^{a}$ Takuya Kawahara ${ }^{c}$ \\ Tetsuro Honda $^{d}$ Kenkei Hasatani ${ }^{e}$ Naohiro Yoshida ${ }^{f}$ Tsutomu Nishidag \\ Tetsuya Sumiyoshi $^{\text {h }}$ Shu Kiyotoki ${ }^{i}$ Takashi lkeya ${ }^{j}$ Masahiro Arai ${ }^{k}$ \\ Nobumi Suzuki ${ }^{a}$ Yosuke Tsuji $^{a}$ Atsuo Yamada ${ }^{a}$ Takashi Kawai ${ }^{b}$ \\ Kazuhiko Koikea \\ aDepartment of Gastroenterology, Graduate School of Medicine, The University of Tokyo, Tokyo, Japan; \\ ${ }^{b}$ Gastroenterological Endoscopy, Tokyo Medical University, Tokyo, Japan; 'Clinical Research Promotion Center, \\ The University of Tokyo Hospital, Tokyo, Japan; 'Department of Gastroenterology, Nagasaki Harbor Medical Center,

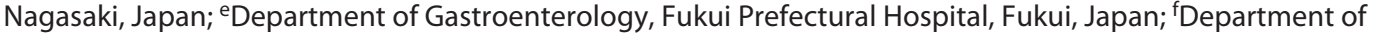 \\ Gastroenterology, Ishikawa Prefectural Central Hospital, Kanazawa-shi, Japan; 9Department of Gastroenterology, \\ Toyonaka Municipal Hospital, Osaka, Japan; ' Department of Gastroenterology, Tonan Hospital, Sapporo-shi, Japan; \\ 'Department of Gastroenterology, Shuto General Hospital, Yamaguchi, Japan; 'Department of Gastroenterology, \\ St. Luke's International Hospital, Tokyo, Japan; 'Department of Gastroenterology, Nerima Hikarigaoka Hospital, \\ Tokyo, Japan
}

\section{Keywords}

Oesophageal cancer · Chemoprevention · Risk stratification · Medication · Aspirin

\footnotetext{
Abstract

Background: Oesophageal cancer comprises 2 different histological variants: oesophageal squamous-cell carcinoma (ESCC) and adenocarcinoma (EAC). While there are multiple therapeutic options for both types, patients with advanced or metastatic oesophageal cancer still suffer from poor prognosis. Aims: The study aimed to examine the association between the risk of oesophageal cancer and medications and to estimate the chemopreventive effects of commonly used drugs. Methods: A multicentre retrospective cohort study was conducted using data from 9 hospital databases of hospitalized patients between 2014 and 2019. The primary outcomes were ESCC and EAC. The association of oesophageal
}

cancer with drug use and clinical factors was evaluated. Odds ratios (ORs) were adjusted for age, sex, Charlson comorbidity index scores, and smoking with/without gastrooesophageal reflux disease. Results: The use of proton pump inhibitors (PPIs) (adjusted OR [aOR] 0.48, $p<0.0001$ ), aspirin (aOR 0.32, $p<0.0001$ ), cyclooxygenase-2 inhibitor (COX2I) (aOR 0.70, $p=0.0005)$, steroid (aOR 0.19, $p<0.0001)$, statin (aOR 0.43, $p<0.0001$ ), and metformin (aOR 0.42, $p<$ 0.0001 ) was associated with a lower risk of ESCC than that in non-use. The use of aspirin (aOR 0.33, $p=0.0006$ ) and steroids (aOR 0.54, $p=0.022$ ) was associated with a lower risk of EAC than that in non-use. Conclusion: COX2Is, statins, metformin, and PPIs could help in prevention of ESCC, and aspirin and steroids may be chemopreventive for both types of oesophageal cancer.

(c) 2021 The Author(s). Published by S. Karger AG, Basel Junya Arai: Orcid ID: 0000-0002-4905-7046.
(C) 2021 The Author(s)

Published by S. Karger AG, Basel

This is an Open Access article licensed under the Creative Commons Attribution-NonCommercial-4.0 International License (CC BY-NC) (http://www.karger.com/Services/OpenAccessLicense), applicable to the online version of the article only. Usage and distribution for commercial purposes requires written permission.
Correspondence to:

Ryota Niikura, niikura-dky@umin.ac.jp

Yoku Hayakawa, yhayakawa-tky@umin.ac.jp 


\section{Introduction}

Oesophageal squamous-cell carcinoma (ESCC) and adenocarcinoma (EAC) are important causes of cancerrelated deaths worldwide [1]. Recently, the incidence of ESCC has increased in Eastern Asia and Eastern and Southern Africa, whereas the incidence of EAC has increased in North America and Europe. Several risk factors for these cancers have been discussed [2]. Smoking and alcohol are the main risk factors for ESCC, whereas gastro-oesophageal reflux disease (GERD) and Barrett's oesophagus, due to gastric acid, are the predominant risk factors for EAC [3-5]. These factors cause chronic inflammation and subsequent DNA damage in the oesophageal epithelium, resulting in cancer development. Given that several drugs have potential anti-inflammatory and anti-DNA damage effects, they may have anti-carcinogenic effects in oesophageal cancer.

In a randomized control study in Western countries, aspirin and esomeprazole, a strong proton pump inhibitor (PPI), prolonged the time to EAC development in patients with Barrett's oesophagus [6]. In a meta-analysis, non-steroidal anti-inflammatory drugs, including aspirin, were associated with a lower risk of ESCC [7]. In another meta-analysis, statins were shown to have a potential chemopreventive effect in both ESCC and EAC [8]. In addition, a population-based cohort study [9] suggested that metformin is associated with a decreased risk of ESCC. However, more comprehensive studies are needed to identify the association between oesophageal cancer and clinical parameters, including medications. Thus, a multicentre retrospective cohort study was performed to evaluate the chemopreventive effects of ESCC and EAC; we found that aspirin, PPI, and several other drugs are associated with lower risk of oesophageal cancers, particularly when these drugs are combined.

\section{Methods}

\section{Study Design, Setting, and Patients}

This retrospective case-control study was performed using a combination of diagnostic procedure databases from 9 hospitals. The study period was from April 2014 to March 2019. The combined databases provided records for all inpatients at Tonan Hospital and for all inpatients and outpatients at the University of Tokyo Hospital, Shuto General Hospital, Fukui Prefectural Hospital, Nerima Hikarigaoka Hospital, St. Luke's International Hospital, Toyonaka Municipal Hospital, Ishikawa Prefectural Central Hospital, and Nagasaki Minato Medical Center. They contained data on diagnosis, comorbidities, and adverse events, recorded using the International Classification of Diseases, tenth revision (ICD-
10), as well as on drugs and procedures, coded using the original Japanese system. This study was approved by the Institutional Review Board of the University of Tokyo Hospital (No. 2019161NI). All patients gave their informed consent prior to their inclusion in the study.

\section{Outcomes and Variables}

The primary outcomes were ESCC and EAC. Oesophageal cancers were defined as ICD-10 codes C150-C159. We assessed the use of PPIs, including omeprazole, lansoprazole, rabeprazole, and esomeprazole; non-steroidal anti-inflammatory drugs, including cyclooxygenase-2 inhibitors (COX2I); aspirin; steroids; statins including pitavastatin, simvastatin, pravastatin, fluvastatin, atorvastatin, and rosuvastatin; fibrates including fenofibrate, bezafibrate, clinofibrate, and clofibrate; other lipid-lowering agents; and metformin. Details of the codes for these medications are shown in online supplementary Table 1 (for all online suppl. material, see www.karger.com/doi/10.1159/000520924). Use of PPIs was defined as a prescription for $>90$ days to eliminate the influence of endoscopic treatment-related PPI use.

We also evaluated the following clinical factors: age, sex, smoking status, and comorbidities. Age was dichotomized as $>70$ and $\leq 70$ years. The following comorbidities (based on ICD-10 codes) were included: atrial fibrillation, acquired immunodeficiency syndrome, arterial thrombosis, carotid disease, cerebrovascular disease, chronic heart failure, chronic kidney disease (stage 5), dementia, diabetes mellitus with or without complications, deep-vein thrombosis, GERD, hemiplegia, dyslipidaemia, ischaemic heart disease, liver disorder (mild/severe), malignancy with or without metastasis, peripheral vascular disease, pulmonary disease, rheumatic disease, transient ischaemic attack, peptic ulcer disease, unstable angina, and valvular disease. The Charlson comorbidity index was calculated using these data [10]. The details of the ICD-10 codes are provided in online supplementary Table 2.

\section{Statistical Analysis}

Univariable and multivariable logistic models were used to estimate the odds ratios (ORs) and 95\% confidence intervals (CIs) for ESCC and EAC. The multivariable logistic models were adjusted for age, sex, smoking, Charlson comorbidity index, and GERD. A subgroup analysis was performed for smoking patients. Additional logistic model analyses were performed to estimate the effect of drug combinations. Statistical significance was set at $p<$ 0.05 . All statistical analyses were performed using SAS software (ver. 9.4; SAS Institute, Cary, NC, USA).

\section{Results}

\section{Patient Characteristics}

A total of 308,793 patients, including 1,911 ESCC, 195 EAC, and 306,687 non-oesophageal cancer patients, were analysed. Patient characteristics are shown in Table $1 . \mathrm{Pa}-$ tients with oesophageal cancer, including ESCC and EAC, had higher proportions of elderly, male, history of smoking, and GERD than those in non-oesophageal cancer patients. 
Table 1. Baseline characteristics (number of patients (\%) or mean \pm standard deviation)

\begin{tabular}{|c|c|c|c|}
\hline Characteristics & $\begin{array}{l}\text { ESCC } \\
(n=1,911)\end{array}$ & $\begin{array}{l}\text { EAC } \\
(n=195)\end{array}$ & $\begin{array}{l}\text { No ESCC or EAC } \\
(n=306,687)\end{array}$ \\
\hline Male & $1,550(81.11)$ & $160(82.05)$ & $149,461(48.73)$ \\
\hline Age $>70$ years & $998(52.22)$ & $80(41.03)$ & $115,128(37.54)$ \\
\hline Smoking & $1,231(64.42)$ & $118(60.51)$ & $99,692(32.51)$ \\
\hline \multicolumn{4}{|l|}{ Medications } \\
\hline PPI & 359 (18.79) & $32(16.41)$ & $27,279(8.89)$ \\
\hline NSAIDs, $N$ & $751(39.30)$ & 77 (39.49) & $106,937(34.87)$ \\
\hline Aspirin & $116(6.07)$ & $10(5.13)$ & $25,708(8.38)$ \\
\hline COX2I (only celecoxib) & $115(5.44)$ & $11(5.64)$ & $16,737(5.46)$ \\
\hline Steroid & $77(4.03)$ & $17(8.72)$ & $27,063(8.82)$ \\
\hline Statin, $N$ & $166(8.69)$ & $28(14.36)$ & $34,035(11.10)$ \\
\hline Strong statin & $141(7.38)$ & $25(12.82)$ & $28,969(9.45)$ \\
\hline Fibrate & $23(1.20)$ & $3(1.54)$ & $2,496(0.81)$ \\
\hline Other lipid-lowering agents & $17(0.89)$ & $5(2.56)$ & 3,947 (1.29) \\
\hline Metformin & $37(1.94)$ & $9(4.62)$ & $7,848(2.56)$ \\
\hline \multicolumn{4}{|l|}{ Comorbidities } \\
\hline Atrial fibrillation & $38(1.99)$ & $2(1.03)$ & $2,657(0.87)$ \\
\hline AIDS & $0(0.00)$ & $0(0.00)$ & $113(0.04)$ \\
\hline Arterial thrombosis & $15(0.78)$ & $1(0.51)$ & $1,443(0.47)$ \\
\hline Carotid disease & $9(0.47)$ & $1(0.51)$ & $862(0.28)$ \\
\hline Cerebrovascular disease & $108(5.65)$ & $6(3.08)$ & $12,721(4.15)$ \\
\hline Chronic heart failure & $108(5.65)$ & $7(3.59)$ & $19,739(6.44)$ \\
\hline Chronic kidney disease <stage 5 & $35(1.83)$ & $7(3.59)$ & $7,595(2.48)$ \\
\hline Chronic kidney disease stage 5 & $4(0.21)$ & $1(0.51)$ & $870(0.28)$ \\
\hline Dementia & $28(1.47)$ & $0(0)$ & $6,710(2.19)$ \\
\hline DM without COMP & $322(16.85)$ & $39(20.00)$ & $34,563(11.27)$ \\
\hline DM with COMP & $39(2.04)$ & $4(2.05)$ & $8,796(2.87)$ \\
\hline DVT & $16(0.84)$ & $2(1.03)$ & $2,538(0.83)$ \\
\hline GERD & $710(37.15)$ & $50(25.64)$ & $23,819(7.77)$ \\
\hline Hemiplegia & $4(0.21)$ & $0(0)$ & $708(0.23)$ \\
\hline HTN & $515(26.95)$ & $40(20.51)$ & $57,461(18.74)$ \\
\hline $\mathrm{HL}$ & $203(10.62)$ & $15(7.69)$ & $30,666(10.00)$ \\
\hline Ischaemic heart diseases & $27(1.41)$ & $0(0)$ & $3,812(1.24)$ \\
\hline LD (mild level) & $129(6.75)$ & $12(6.15)$ & $10,327(3.37)$ \\
\hline LD (severe level) & $16(0.84)$ & $0(0)$ & $1,255(0.41)$ \\
\hline Malignancy without metastasis & $553(28.94)$ & $55(28.21)$ & $19,787(6.45)$ \\
\hline Malignancy with metastasis & $237(12.40)$ & $18(9.23)$ & $9,631(3.14)$ \\
\hline PVDs & $34(1.78)$ & $2(1.03)$ & $5,299(1.73)$ \\
\hline PD & $129(6.75)$ & $11(5.64)$ & $14,447(4.71)$ \\
\hline RDs & $6(0.31)$ & $2(1.03)$ & $1,480(0.48)$ \\
\hline TIA & $3(0.16)$ & $0(0)$ & $285(0.09)$ \\
\hline PUDs & $671(35.11)$ & $74(37.95)$ & $24,146(7.87)$ \\
\hline UAGs & $138(7.22)$ & $19(9.74)$ & $14,261(4.65)$ \\
\hline VDs & $20(1.05)$ & $6(3.08)$ & $3,768(1.23)$ \\
\hline Charlson comorbidities index & $2.22 \pm 2.57$ & $1.95 \pm 2.18$ & $0.84 \pm 1.65$ \\
\hline
\end{tabular}

ESCC, oesophageal squamous-cell carcinoma; EAC, oesophageal adenocarcinoma; DM, diabetes mellitus; NSAIDs, non-steroidal anti-inflammatory drugs; COX2I, cyclooxygenase-2 inhibitor; PPI, proton pump inhibitor; GERD, gastro-oesophageal reflux disease; COMP, complications; DVT, deep-vein thrombosis; HTN, hypertension; $\mathrm{HL}$, hyperlipidaemia; $L D$, liver disorder; PVD, peripheral vascular disease; $P D$, pulmonary disease; $R D$, rheumatic diseases; TIA, transient ischaemic attack; PUD, peptic ulcer disease; UAG, unstable angina diseases; VD, valvular diseases; AIDS, acquired immunodeficiency syndrome. 
Table 2. Clinical factors associated with $\operatorname{ESCC}(n=308,598)$

\begin{tabular}{|c|c|c|c|c|c|}
\hline Factor & $\begin{array}{l}\text { ESCC } \\
(n=1,911)\end{array}$ & $\begin{array}{l}\text { No ESCC } \\
(n=306,687)\end{array}$ & $\begin{array}{l}\text { Crude OR } \\
(95 \% \mathrm{Cl})\end{array}$ & $\begin{array}{l}\text { Adjusted OR } \\
(95 \% \mathrm{Cl})\end{array}$ & $p$ value \\
\hline Female & $361(0.23)$ & 157,226 (99.77) & 1 & 1 & \\
\hline Age $\leq 70$ & $913(0.47)$ & 191,559 (99.53) & 1 & 1 & \\
\hline Age $>70$ & $998(0.86)$ & $115,128(99.14)$ & $1.82(1.66-1.99)$ & $1.15(1.13-1.17)$ & $<0.0001$ \\
\hline Non-smoking & $680(0.33)$ & 206,995 (99.67) & 1 & 1 & \\
\hline PPI & $297(1.08)$ & $27,279(98.92)$ & $1.89(1.67-2.14)$ & $0.48(0.42-0.56)$ & $<0.0001$ \\
\hline No NSAIDs & $1,160(0.58)$ & $199,750(99.42)$ & 1 & 1 & \\
\hline NSAIDs & $751(0.70)$ & $106,937(99.30)$ & $1.21(1.10-1.33)$ & $0.80(0.72-0.88)$ & $<0.0001$ \\
\hline No aspirin & $1,795(0.63)$ & 280,979 (99.37) & 1 & 1 & \\
\hline Aspirin & $116(0.45)$ & $25,708(99.55)$ & $0.71(0.59-0.85)$ & $0.32(0.26-0.39)$ & $<0.0001$ \\
\hline No statin & $1,745(0.64)$ & $272,652(99.36)$ & 1 & 1 & \\
\hline Statin & $166(0.49)$ & 34,035 (99.51) & $0.76(0.65-0.89)$ & $0.43(0.37-0.51)$ & $<0.0001$ \\
\hline No strong statin & $1,770(0.63)$ & 277,718 (99.37) & 1 & 1 & \\
\hline Strong statin & $141(0.48)$ & 28,969 (99.52) & $0.76(0.64-0.91)$ & $0.41(0.35-0.49)$ & $<0.0001$ \\
\hline No fibrate & $1,888(0.62)$ & 304,191 (99.38) & 1 & 1 & \\
\hline Fibrate & $23(0.91)$ & 2,496 (99.09) & $1.49(0.98-2.24)$ & $0.89(0.59-1.36)$ & 0.595 \\
\hline No other lipid-lowering agent & $1,894(0.62)$ & $302,740(99.38)$ & 1 & 1 & \\
\hline Other lipid-lowering agents & $17(0.43)$ & $3,947(99.57)$ & $0.69(0.43-1.11)$ & $0.40(0.25-0.64)$ & 0.0002 \\
\hline No metformin & $1,874(0.62)$ & $298,839(99.38)$ & 1 & 1 & \\
\hline Metformin & $37(0.47)$ & $7,848(99.53)$ & $0.75(0.54-1.04)$ & $0.42(0.30-0.58)$ & $<0.0001$ \\
\hline Non-Af & $1,873(0.61)$ & $304,030(99.31)$ & 1 & 1 & \\
\hline Af & $38(1.41)$ & $2,657(98.59)$ & $2.32(1.68-3.21)$ & $1.02(0.73-1.42)$ & 0.919 \\
\hline Non-CHF & $1,803(0.62)$ & $286,948(99.38)$ & 1 & 1 & \\
\hline $\mathrm{CHF}$ & $108(0.54)$ & $19,739(99.46)$ & $0.87(0.72-1.06)$ & $0.35(0.29-0.43)$ & $<0.0001$ \\
\hline Non-CKD <stage 5 & $1,876(0.62)$ & $299,092(99.38)$ & 1 & 1 & \\
\hline CKD < stage 5 & $35(0.46)$ & $7,595(99.54)$ & $0.74(0.53-1.03)$ & $0.34(0.24-0.48)$ & $<0.0001$ \\
\hline Non-CKD stage 5 & $1,907(0.62)$ & $305,817(99.38)$ & 1 & 1 & \\
\hline CKD stage 5 & $4(0.46)$ & 870 (99.54) & $0.74(0.28-1.97)$ & $0.20(0.073-0.53)$ & 0.0013 \\
\hline Non-dementia & $1,883(0.62)$ & $299,977(99.38)$ & 1 & 1 & \\
\hline Dementia & $28(0.42)$ & $6,710(99.58)$ & $0.67(0.46-0.97)$ & $0.48(0.33-0.70)$ & 0.0002 \\
\hline Non-DM (COMP-) & $1,589(0.58)$ & $272,124(99.42)$ & 1 & 1 & \\
\hline DM (COMP-) & $322(0.92)$ & $34,563(99.08)$ & $1.60(1.42-1.80)$ & $0.64(0.57-0.73)$ & $<0.0001$ \\
\hline Non-DM (COMP+) & $1,872(0.62)$ & $297,891(99.38)$ & 1 & 1 & \\
\hline $\mathrm{DM}(\mathrm{COMP}+)$ & $39(0.44)$ & $8,796(99.56)$ & $0.71(0.51-0.97)$ & $0.25(0.18-0.34)$ & $<0.0001$ \\
\hline Non-DVT & $1,895(0.62)$ & $304,149(99.38)$ & 1 & 1 & \\
\hline DVT & $16(0.63)$ & $2,538(99.37)$ & $1.01(0.62-1.66)$ & $0.59(0.36-0.97)$ & 0.036 \\
\hline Non-GERD & $1,201(0.42)$ & 282,868 (99.58) & 1 & 1 & \\
\hline GERD & $710(2.89)$ & $23,819(97.11)$ & 7.02 (6.39-7.71) & $4.44(4.01-4.91)$ & $<0.0001$ \\
\hline Non-hemiplegia & $1,907(0.62)$ & $305,979(99.42)$ & 1 & 1 & \\
\hline Hemiplegia & $4(0.56)$ & $708(99.44)$ & $0.91(0.34-2.43)$ & $0.44(0.16-1.20)$ & 0.1080 \\
\hline
\end{tabular}


Table 2 (continued)

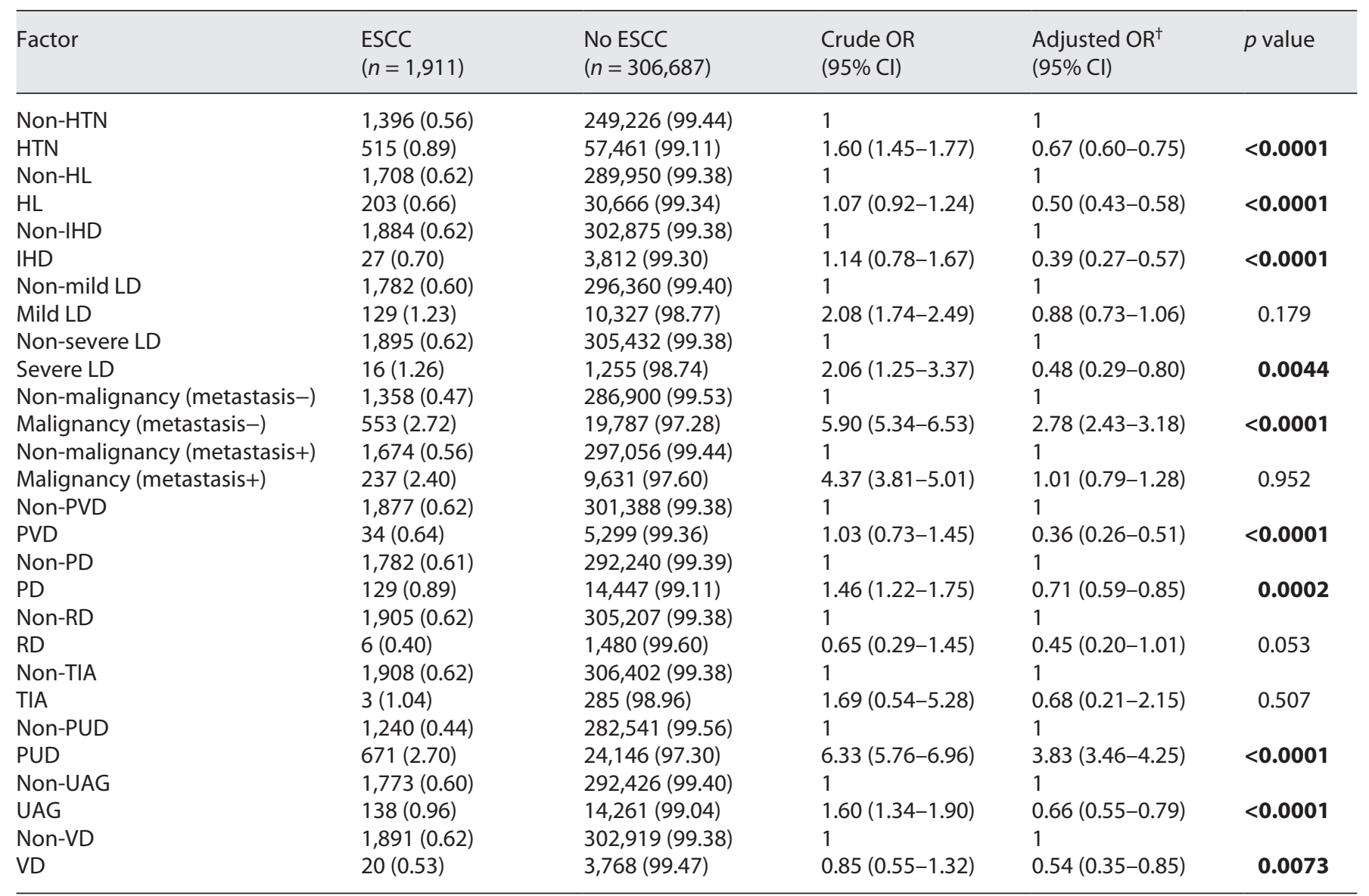

ESCC, oesophageal squamous-cell carcinoma; OR, odds ratio; $\mathrm{Cl}$, confidence interval; NSAID, non-steroidal anti-inflammatory drug; COX2I, cyclooxygenase-2 inhibitor; PPI, proton pump inhibitor; Af, atrial fibrillation; AIDS, acquired immunodeficiency syndrome; AT, arterial thrombosis; $C D$, carotid disease; CVD, cerebrovascular disease; CHF, chronic heart failure; CKD, chronic kidney disease; DM, diabetes mellitus; COMP, complications; DVT, deep-vein thrombosis; GERD, gastro-oesophageal reflux disease; $\mathrm{HTN}$, hypertension; $\mathrm{HL}$, hyperlipidaemia; LD, liver disorder; PE, pulmonary embolism; PVD, peripheral vascular disease; PD, pulmonary disease; RD, rheumatic diseases; TIA, transient ischaemic attack; PUD, peptic ulcer disease; UAG, unstable angina diseases; VD, valvular diseases. ${ }^{\dagger}$ OR adjusted for age $>70$ years, sex, smoking, GERD, and Charlson comorbidity index.

\section{Factors Associated with ESCC}

The factors associated with ESCC are listed in Table 2. The use of PPI (adjusted OR [aOR] 0.48, 95\% CI $0.42-0.56$ ), aspirin (aOR 0.32 , 95\% CI $0.26-0.39$ ), COX2I (aOR 0.70, 95\% CI 0.57-0.86), steroid (aOR $0.19,95 \%$ CI $0.15-0.24$ ), statin (aOR $0.43,95 \%$ CI $0.37-$ 0.51 ), and metformin (aOR 0.42 , 95\% CI $0.30-0.58$ ) were associated with a lower risk of oesophageal SCC than that in non-use. These results were similar in the subgroup of smoking patients (Table 3 ) and in another logistic model analysis adjusted for propensity score calculated by age, sex, smoking, and comorbidity (online suppl. Table 3 ).

\section{Factors Associated with EAC}

The factors associated with the EAC are shown in Table 4 . The use of aspirin (aOR $0.33,95 \%$ CI $0.17-0.62$ ) and steroids (aOR 0.54, 95\% CI 0.32-0.91) was associated with a lower risk of EAC than that in non-use. These results were similar in the subgroup of smoking patients (Table 5) and in another logistic model analysis adjusted for propensity score calculated by age, sex, smoking, and comorbidity (online suppl. Table 3 ).

\section{Drug Combination Model for ESCC and EAC}

The drug combination models for ESCC and EAC are shown in Table 6. Drug combinations of PPI and aspirin 
Table 3. Clinical factors associated with ESCC in smoking patients $(n=100,923)$

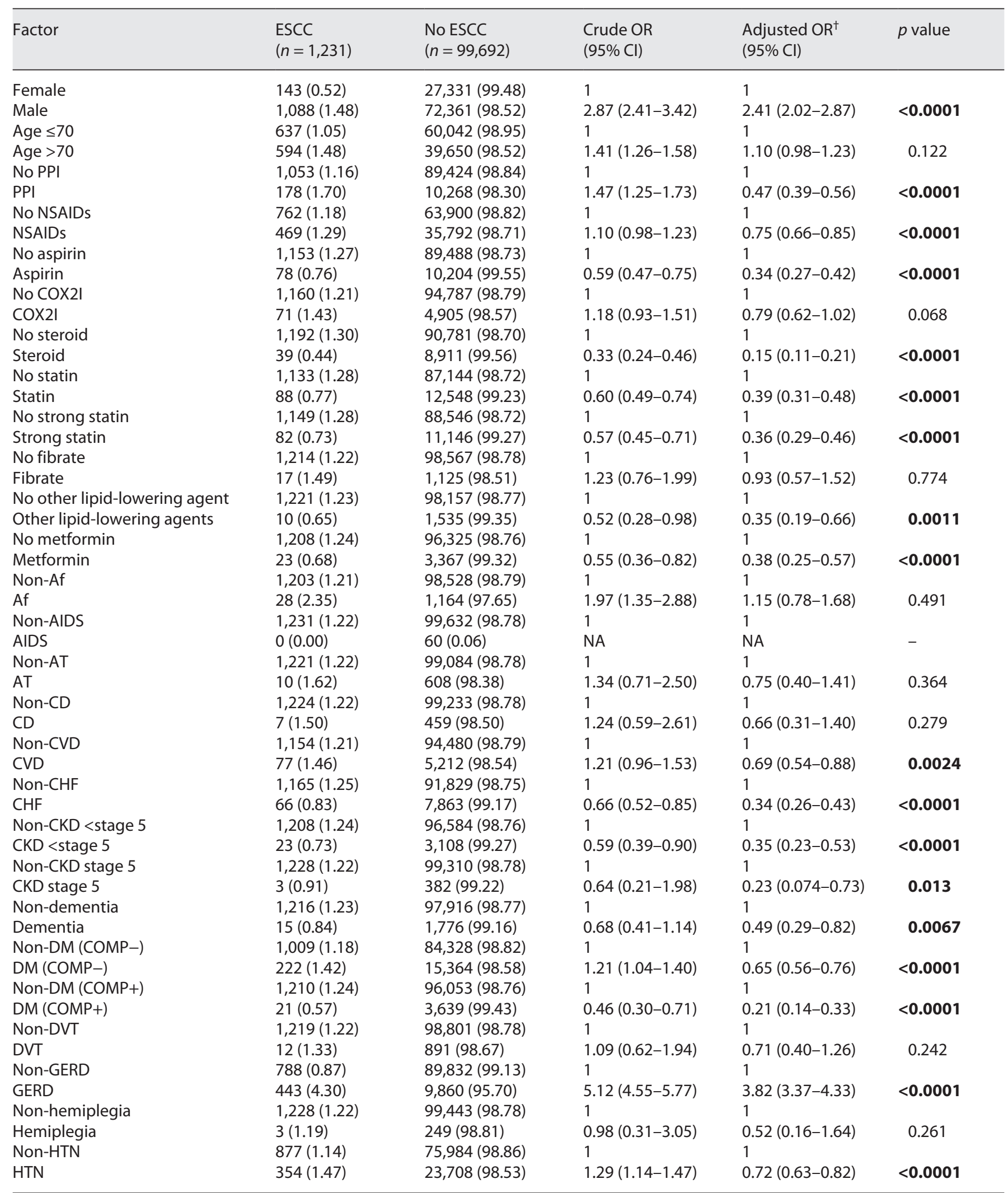


Table 3 (continued)

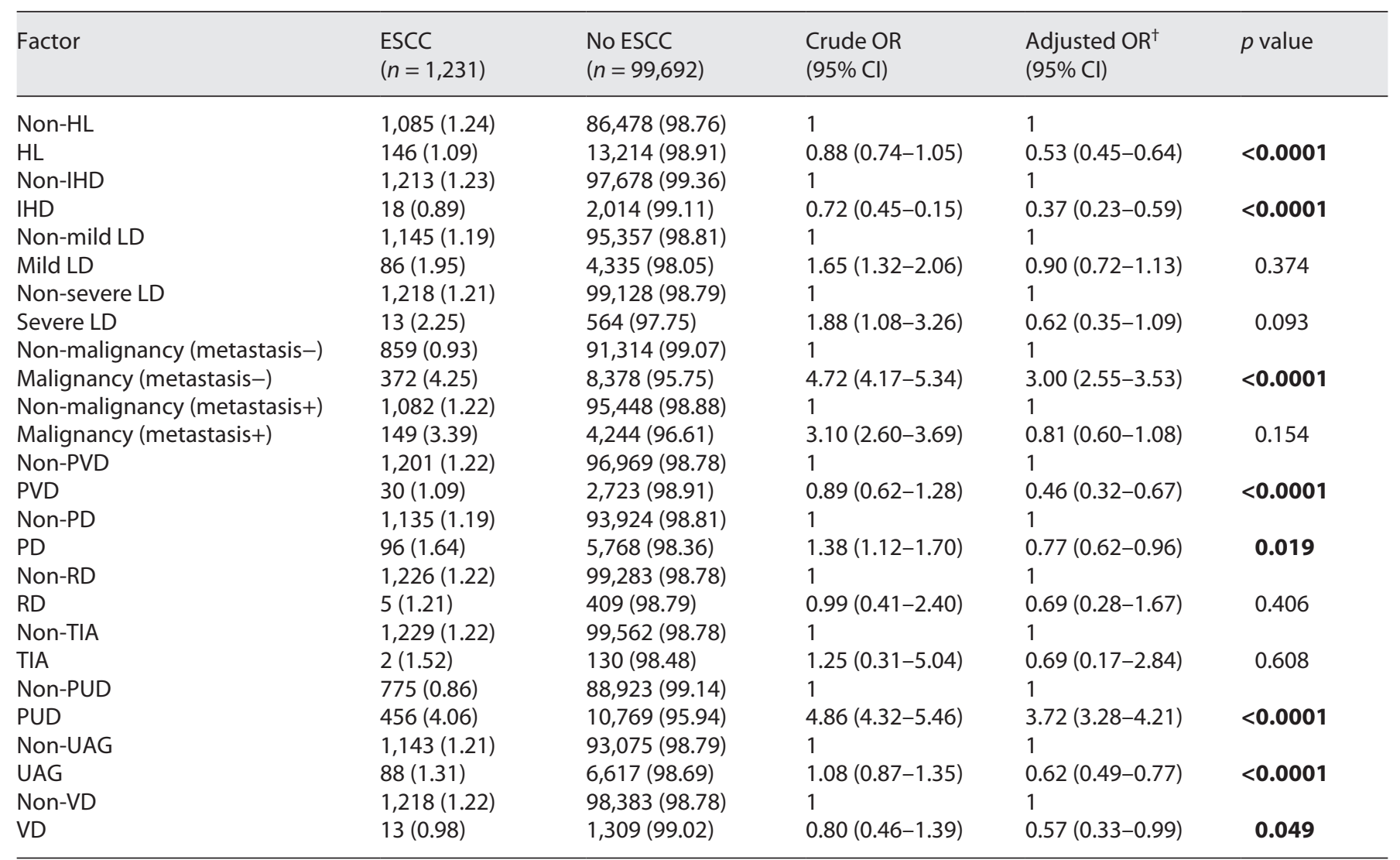

ESCC, oesophageal squamous-cell carcinoma; OR, odds ratio; $\mathrm{Cl}$, confidence interval; NSAID, non-steroidal anti-inflammatory drug; COX2I, cyclooxygenase-2 inhibitor; PPI, proton pump inhibitor; Af, atrial fibrillation; AIDS, acquired immunodeficiency syndrome; AT, arterial thrombosis; $C D$, carotid disease; CVD, cerebrovascular disease; CHF, chronic heart failure; CKD, chronic kidney disease; DM, diabetes mellitus; COMP, complications; DVT, deep-vein thrombosis; GERD, gastro-oesophageal reflux disease; HTN, hypertension; HL, hyperlipidaemia; LD, liver disorder; PE, pulmonary embolism; PVD, peripheral vascular diseases; PD, pulmonary disease; RD, rheumatic diseases; TIA, transient ischaemic attack; PUD, peptic ulcer disease; UAG, unstable angina diseases; VD, valvular diseases. ${ }^{\dagger}$ OR adjusted for age $>70$ years, sex, GERD, and Charlson comorbidity index (except for the variable analysed).

(aOR 0.26, 95\% CI 0.19-0.34), COX2I (aOR 0.51, 95\% CI $0.36-0.72$ ), steroids (aOR 0.26, 95\% CI 0.20-0.35), statin (aOR 0.31, 95\% CI 0.24-0.41), and metformin (aOR 0.32, 95\% CI 0.18-0.57) were associated with a lower risk of ESCC than that in PPI without these medications. A combination of PPI and aspirin (aOR 0.27, 95\% CI 0.11-0.68) was associated with a lower risk of EAC compared with PPI without aspirin.

\section{Discussion}

In this multicentre retrospective cohort study, we found that the use of PPI, aspirin, COX2I, steroid, statin, and metformin was associated with a lower risk of ESCC, while aspirin and steroids were associated with a lower risk of EAC.

In line with the previous studies, being male is one of the main risk factors of both ESCC and EAC. One likely reason for this is that male patients tend to have more tobacco and alcohol consumption than female patients, both of which are clear risk factors of ESCC and EAC. Moreover, female hormones are reported to be preventive for ESCC and EAC in several previous studies [1113]. Indeed, subgroup analysis according to age classes showed that the OR of male compared to female for EAC steadily decreased with ageing, while that for ESCC continuously increased (online suppl. Table 4). Furthermore, menopausal hormone drugs tended to be associated with a lower risk of both ESCC and EAC for female patients 
Table 4. Clinical factors associated with EAC $(n=306,682)$

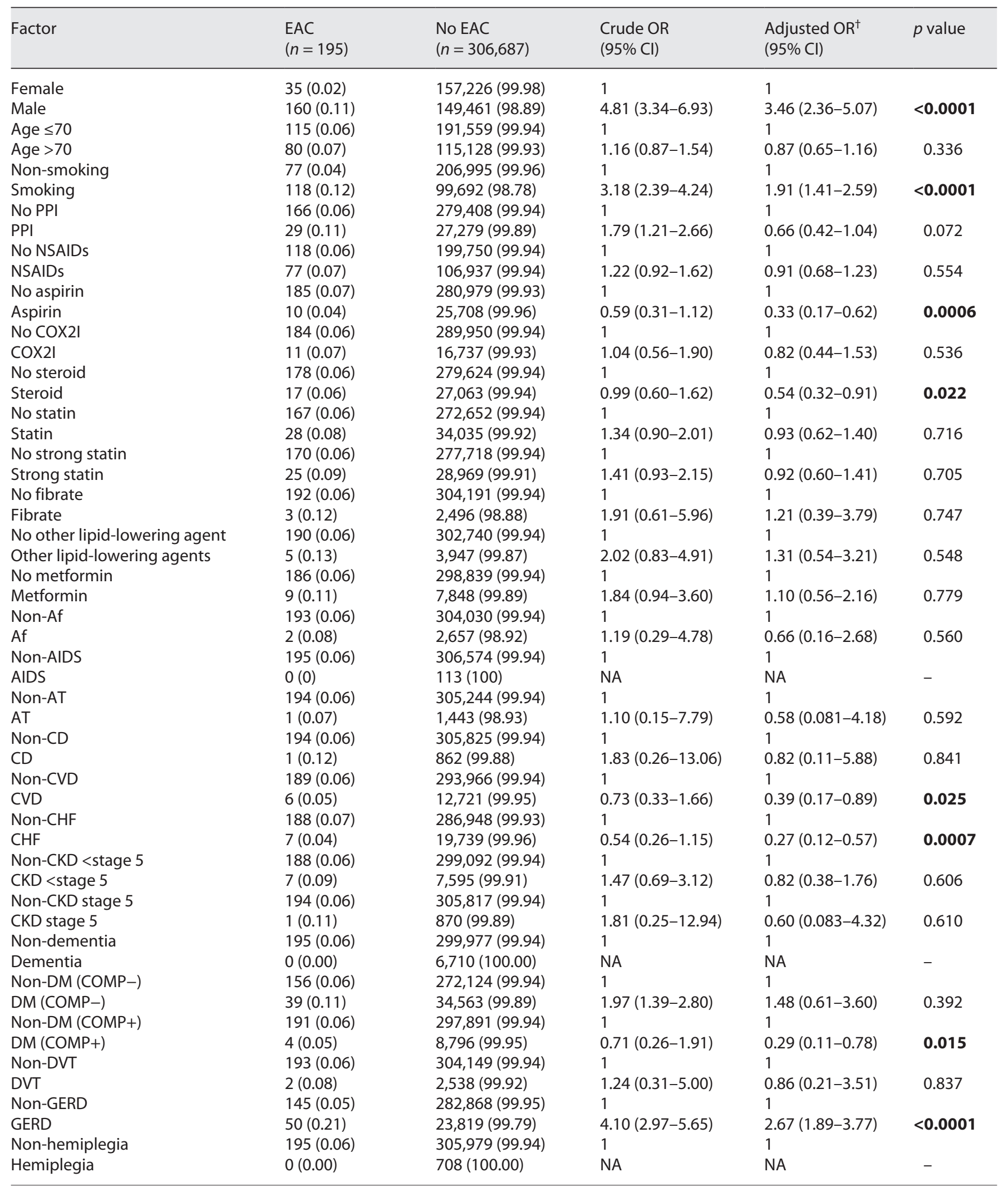


Table 4 (continued)

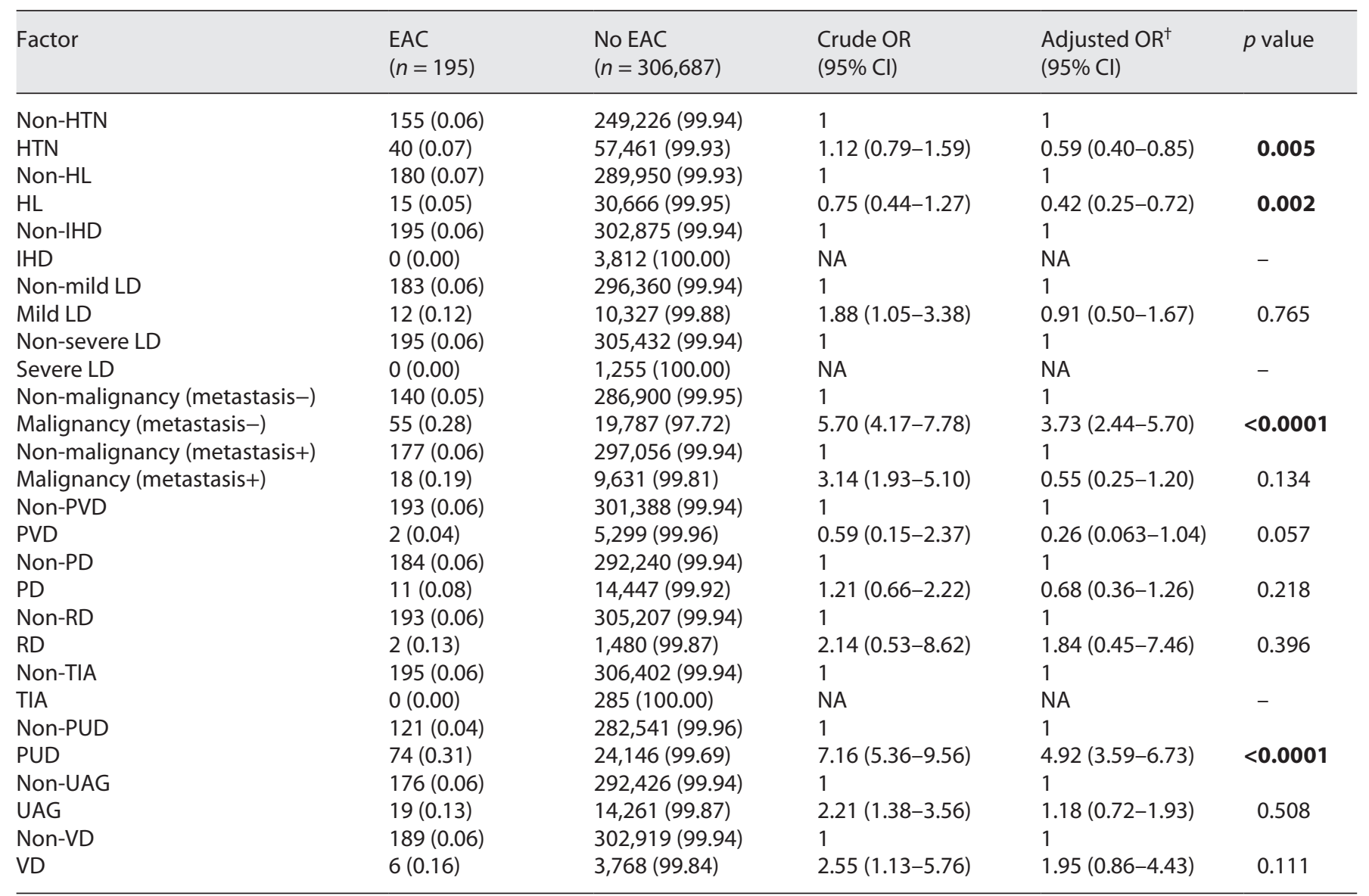

EAC, oesophageal adenocarcinoma; OR, odds ratio; Cl, confidence interval; NSAID, non-steroidal anti-inflammatory drug; COX2I, cyclooxygenase-2 inhibitor; PPI, proton pump inhibitor; Af, atrial fibrillation; AIDS, acquired immunodeficiency syndrome; AT, arterial thrombosis; CD, carotid disease; CVD, cerebrovascular disease; CHF, chronic heart failure; CKD, chronic kidney disease; DM, diabetes mellitus; COMP, complications; DVT, deep-vein thrombosis; GERD, gastro-oesophageal reflux disease; HTN, hypertension; HL, hyperlipidaemia; LD, liver disorder; $P E$, pulmonary embolism; PVD, peripheral vascular diseases; $P D$, pulmonary disease; $R D$, rheumatic diseases; TIA, transient ischaemic attack; PUD, peptic ulcer disease; UAG, unstable angina diseases; VD, valvular diseases. ${ }^{\dagger}$ OR adjusted for age $>70$ years, sex, smoking, GERD, and Charlson comorbidity index (except for the variable analysed).

with age $>50$ in our cohort (online suppl. Table 5). Therefore, female hormones may be preventive for oesophageal cancers, particularly for EAC.

In our study, PPI use was associated with a lower risk of ESCC but not with EAC. Since PPIs are often used for GERD patients and GERD is likely a confounder for EAC risk, we performed subgroup analyses (online suppl. Table 6). Interestingly, PPI use was associated with a lower risk of both ESCC and EAC in patients with GERD. These results are compatible with those of a previous study that showed that the PPI was chemopreventive against EAC in patients with Barrett's oesophagus [6]. PPIs strongly reduce gastric acid reflux [14] and may contribute to re- ducing acid reflux-related carcinogenesis in patients with GERD and Barrett's oesophagus.

Although the PPI was shown to be associated with lower risk of both ESCC and EAC, several adverse effects by long-term PPI use should be noted. The recent study showed that long-term PPI use was associated with a higher risk of gastric cancer $[15,16]$, potentially due to the worsened gastric atrophy by PPIs [17, 18]. Moreover, longterm PPI use causes consequently a high level of serum gastrin, which may increase gastric carcinoid development [19]. Indeed, overexpression of amidated gastrin and/or long-term PPI treatment promote epithelial proliferation and tumorigenesis in the stomach and even the oesopha- 
Table 5. Clinical factors associated with EAC in smoking patients $(n=99,810)$

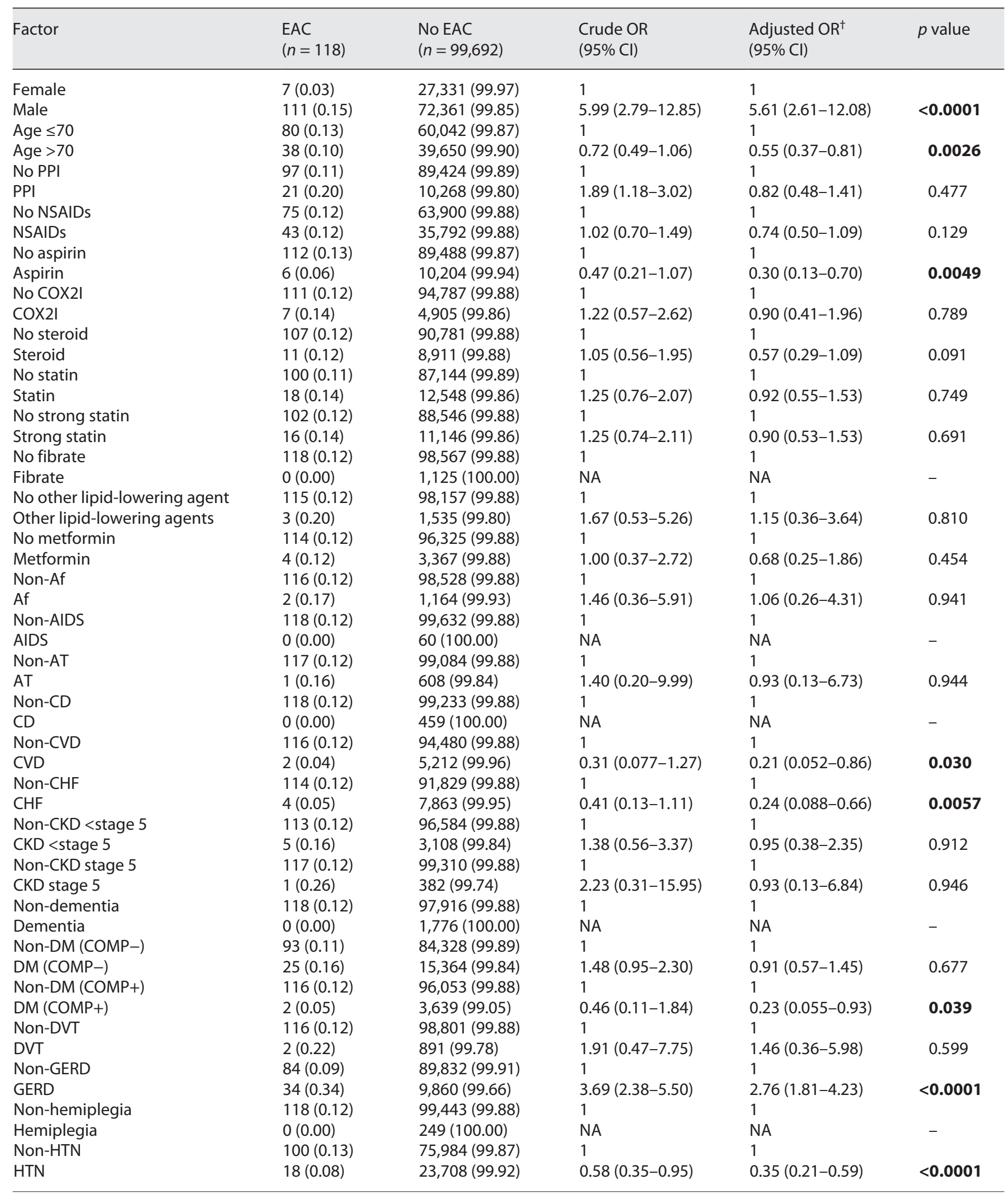


Table 5 (continued)

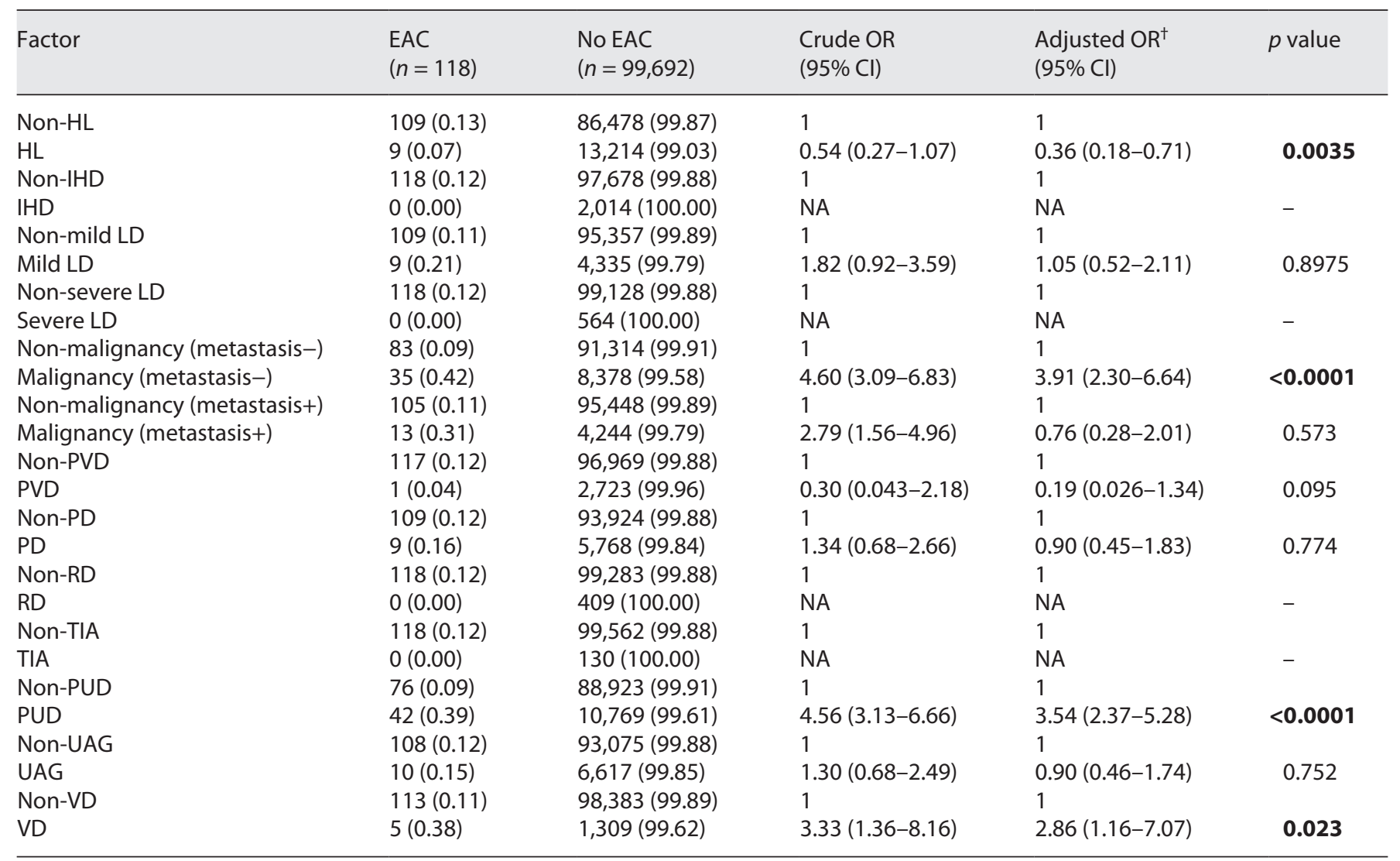

EAC, oesophageal adenocarcinoma; OR, odds ratio; $\mathrm{Cl}$, confidence interval; NSAID, non-steroidal anti-inflammatory drug; COX2I, cyclooxygenase-2 inhibitor; PPI, proton pump inhibitor; Af, atrial fibrillation; AIDS, acquired immunodeficiency syndrome; AT, arterial thrombosis; CD, carotid disease; CVD, cerebrovascular disease; CHF, chronic heart failure; CKD, chronic kidney disease; DM, diabetes mellitus; COMP, complications; DVT, deep-vein thrombosis; GERD, gastro-oesophageal reflux disease; HTN, hypertension; HL, hyperlipidaemia; LD, liver disorder; PE, pulmonary embolism; PVD, peripheral vascular diseases; PD, pulmonary disease; RD, rheumatic diseases; TIA, transient ischaemic attack; PUD, peptic ulcer disease; UAG, unstable angina diseases; VD, valvular diseases. ${ }^{\dagger}$ OR adjusted for age $>70$ years, sex, GERD, and Charlson comorbidity index (except for the variable analysed).

gus in mice [20-23]. PPIs also cause gut dysbiosis by suppressing acid secretion, which may affect the development of gastrointestinal cancers and other diseases [24]. Given that the study period in our study was relatively short, we might underestimate the risk for PPI use. Further study is needed to confirm the recommended duration of PPI use for the chemoprevention of ESCC and EAC.

In our study, the use of aspirin, a COX2I, and steroids was associated with a lower risk of ESCC. Aspirin and steroids were also associated with a lower risk of EAC, and COX2Is tend to be related to a lower risk of EAC, but the difference was not statistically significant. Such differences in the results between ESCC and EAC might be due to the sample size of cancer patients as EAC cases accounted for only $10 \%$ of all cancer patients in the present study.
Oesophageal tumorigenesis was induced by chronic inflammation resulting from various factors, mainly including smoking, alcohol, and GERD, through interleukin-6/ STAT3, nuclear factor-kappaB, and COX2 pathway [25]. Therefore, these anti-inflammatory reagents may suppress chronic inflammation, mainly through downregulation of COX2, which results in decreased cancer risk [26].

In our study, statin use was also associated with a lower risk of ESCC but not with EAC. Several previous studies have shown the chemopreventive effect of statins against EAC $[8,27]$. This discrepancy might be due to the small sample size of the EAC in our study. Moreover, the use of metformin was associated with a lower risk of ESCC but not EAC. This result is compatible with those of previous studies $[9,28]$. The different effects of metformin on ESCC 
Table 6. Drug combination model for ESCC and EAC

\begin{tabular}{|c|c|c|c|c|}
\hline Drug combination & $\begin{array}{l}\text { ESCC } \\
\text { adjusted OR }{ }^{\dagger}(95 \% \text { Cl) }\end{array}$ & $p$ value & $\begin{array}{l}\text { EAC } \\
\text { adjusted } \mathrm{OR}^{\dagger}(95 \% \mathrm{Cl})\end{array}$ & $p$ value \\
\hline PPI & 1 & & 1 & \\
\hline PPI and aspirin & $0.26(0.19-0.34)$ & $<0.0001$ & $0.27(0.11-0.68)$ & 0.006 \\
\hline PPI & 1 & & 1 & \\
\hline $\mathrm{PPI}$ and COX2I & $0.51(0.36-0.72)$ & 0.0001 & $0.14(0.02-1.04)$ & 0.055 \\
\hline PPI & 1 & & 1 & \\
\hline PPI and steroid & $0.26(0.20-0.35)$ & $<0.0001$ & $0.49(0.24-1.03)$ & 0.061 \\
\hline PPI & 1 & & 1 & \\
\hline PPI and statin & $0.31(0.24-0.41)$ & $<0.0001$ & $0.65(0.33-1.27)$ & 0.209 \\
\hline PPI & 1 & & 1 & \\
\hline PPI and metformin & $0.32(0.18-0.57)$ & 0.0001 & $0.26(0.04-1.89)$ & 0.183 \\
\hline
\end{tabular}

and EAC may suggest the presence of distinct molecular pathways in these cancer variants, but further studies are required to understand the exact biology.

The effect of drug exposure in a subgroup of smoking patients using other logistic models was estimated (Tables $3,5)$. In both ESCC and EAC, the effect of drug exposure on cancer was similar in all patient groups. These drugs have potential anti-carcinogenic effects even in patients at high risk of oesophageal cancer. Furthermore, our additional analyses using other logistic models showed that drug combinations of PPI with several drugs were associated with a lower risk of ESCC and EAC (Table 6).

Our study had several strengths. Firstly, we evaluated associations between the use of various drugs and oesophageal cancer in a large number of patients, including both ESCC and EAC. Secondly, this study had multicentre cohort data. Nevertheless, the study has several limitations. Firstly, this was a retrospective study. Secondly, information on risk factors, including diet, body mass index, and family history, was limited to our database. Thirdly, drugs prescribed outside the study centre, including over-the-counter ones, could not be evaluated. Fourthly, the selection bias was concerned as our population was high-volume centre patients in Japan. We speculated that our population had potentially higher comorbidities than in general Japanese population and that risk assessment might be overestimated for healthy population. In addition, smoking exposure and alcohol consumption and alcohol-degrading enzyme activity might differ between Asian population including Japanese and Western country population. Therefore, our results might reduce generalizability for healthy population and Western country population. Finally, unknown confounders and misclassification might not be arranged precisely due to the study design. Similar studies with detailed database were desirable in the future.

\section{Conclusion}

The use of PPI, aspirin, COX2Is, steroids, statins, and metformin was associated with a lower risk of ESCC, even in the smoking population. Aspirin and steroids were also associated with a lower risk of EAC. PPI use was associated with a lower risk of both ESCC and EAC in patients with GERD. These drugs may be potential candidates for the prevention of oesophageal cancer, while long-term effects by these drugs should be carefully assessed in larger and longer cohorts in future.

\section{Acknowledgments}

All the authors approved the final version of the article, including the authorship list.

\section{Statement of Ethics}

The study was conducted according to the guidelines of the Declaration of Helsinki and received ethics approval from the institutional review boards of the University of Tokyo (ID: 2058-[2]). Written informed consent was obtained from the patients. 


\section{Conflict of Interest Statement}

The authors have no conflict of interest to declare.

\section{Funding Sources}

Declaration of funding interests: This study was supported by KAKENHI Grants-in-Aid for Scientific Research (Grant No. $20 \mathrm{~K} 08375$ [R. Niikura] and 17H05081 [Y. Hayakawa]), P-CREATE from AMED, and the Advanced Research and Development Programs for Medical Innovation (PRIME; Y. Hayakawa). The funding agencies had no role in the design of the study, data collection or analysis, decision to publish, or preparation of the manuscript.

\section{Author Contributions}

J.A., R.N., and Y.H. contributed to the study design. J.A. wrote the initial draft of the manuscript. J.A., R.N., and T.K.1 analysed the data. T.H., K.H., N.Y., T.N., T.S., S.K., T.I., M.A., N.S., Y.T., A.Y., T.K.2, and K.K. provided guidance regarding the study design and contributed to manuscript editing.

\section{Data Availability Statement}

All data generated or analysed during this study are included in this article. Further enquiries can be directed to the corresponding author.

\section{References}

1 Simard EP, Ward EM, Siegel R, Jemal A. Cancers with increasing incidence trends in the United States: 1999 through 2008 CA. Cancer J Clin. 2012;62:118-28.

2 Torre LA, Bray F, Siegel RL, Ferlay J, LortetTieulent J, Jemal A. Global cancer statistics, 2012. CA Cancer J Clin. 2015;65:87-108.

3 Domper Arnal MJ, Ferrández Arenas Á, Lanas Arbeloa Á. Esophageal cancer: risk factors, screening and endoscopic treatment in western and eastern countries. World J Gastroenterol. 2015;21:7933-43.

4 Spechler SJ, Souza RF. Barrett's esophagus. N Engl J Med. 2014;371(9):836-45.

5 Hayakawa Y, Sethi N, Sepulveda AR, Bass AJ, Wang TC. Oesophageal adenocarcinoma and gastric cancer: should we mind the gap? Nat Rev Cancer. 2016;16:305-18.

6 Jankowski JAZ, de Caestecker J, Love SB, Reilly G, Watson P, Sanders S, et al. Esomeprazole and aspirin in Barrett's oesophagus (AspECT): a randomised factorial trial. Lancet. 2018;392:400-8.

7 Sun L, Yu S. Meta-analysis: non-steroidal anti-inflammatory drug use and the risk of oesophageal squamous cell carcinoma. Dis Esophagus. 2011;24:544-9.

8 Singh S, Singh AG, Singh PP, Murad MH, Iyer PG. Statins are associated with reduced risk of oesophageal cancer, particularly in patients with Barrett's esophagus: a systematic review and meta-analysis. Clin Gastroenterol Hepatol. 2013;11:620-9.

9 Wang QL, Santoni G, Ness-Jensen E, Lagergren J, Xie SH. Association between metformin use and risk of esophageal squamous cell carcinoma in a Population-Based Cohort Study. Am J Gastroenterol. 2020;115:73-8.

10 Charlson ME, Pompei P, Ales KL, MacKenzie CR. A new method of classifying prognostic comorbidity in longitudinal studies: development and validation. J Chronic Dis. 1987;40: 373-83.
11 Zhang GQ, Chen JL, Luo Y, Mathur MB, Anagnostis P, Nurmatov U, et al. Menopausal hormone therapy and women's health: an umbrella review. PLoS Med. 2021;18(8): e1003731.

12 McCarthy CE, Field JK, Marcus MW. Age at menopause and hormone replacement therapy as risk factors for head and neck and oesophageal cancer (review). Oncol Rep. 2017; 38(4):1915-22.

13 Zhu Y, Yue D, Yuan B, Zhu L, Lu M. Reproductive factors are associated with oesophageal cancer risk: results from a meta-analysis of observational studies. Eur J Cancer Prev. 2017;26(1):1-9.

14 Fass R, Hell RW, Garewall HS, Martinez P, Pulliam G, Wendel C, et al. Correlation of oesophageal acid exposure with Barrett's oesophagus length. Gut. 2001;48:310.

15 Cheung KS, Chan EW, Wong AYS, Chen L, Wong ICK, Leung WK. Long-term proton pump inhibitors and risk of gastric cancer development after treatment for Helicobacter pylori: a population-based study. Gut. 2018; 67:28-35.

16 Niikura R, Hayakawa Y, Hirata Y, Yamada A, Fujishiro M, Koike K. Long-term proton pump inhibitor use is a risk factor of gastric cancer after treatment for helicobacter pylori: a retrospective cohort analysis. Gut. 2018; 67(10):1908-10.

17 Kuipers EJ, Nelis GF, Klinkenberg-Knol EC, Snel P, Goldfain D, Kolkman JJ, et al. Cure of helicobacter pylori infection in patients with reflux oesophagitis treated with long term omeprazole reverses gastritis without exacerbation of reflux disease: results of a randomised controlled trial. Gut. 2004;53:12-20.

18 Niikura R, Hayakawa Y, Hirata Y, Ogura K, Fujishiro M, Yamada A, et al. The reduction in gastric atrophy after helicobacter pylori eradication is reduced by treatment with inhibitors of gastric acid secretion. Int J Mol Sci. 2019;20(8):1913.
19 McCarthy DM. Proton pump inhibitor use, hypergastrinemia, and gastric carcinoidswhat is the relationship? Int J Mol Sci. 2020; 21(2):662.

20 Wang TC, Dangler CA, Chen D, Goldenring JR, Koh T, Raychowdhury R, et al. Synergistic interaction between hypergastrinemia and helicobacter infection in a mouse model of gastric cancer. Gastroenterology. 2000;118(1):36-47.

21 Hayakawa Y, Chang W, Jin G, Wang TC. Gastrin and upper GI cancers. Curr Opin Pharmacol. 2016;31:31-7.

22 Lee Y, Urbanska AM, Hayakawa Y, Wang H, $\mathrm{Au}$ AS, Luna AM, et al. Gastrin stimulates a cholecystokinin-2-receptor-expressing cardia progenitor cell and promotes progression of Barrett's-like esophagus. Oncotarget. 2017; 8(1):203-14

23 Sheng W, Malagola E, Nienhüser H, Zhang Z, Kim W, Zamechek L, et al. Hypergastrinemia expands gastric ECL cells through CCK2R+ progenitor cells via ERK activation. Cell Mol Gastroenterol Hepatol. 2020;10(2):434-49.

24 Bruno G, Zaccari P, Rocco G, Scalese G, Panetta $\mathrm{C}$, Porowska $\mathrm{B}$, et al. Proton pump inhibitors and dysbiosis: current knowledge and aspects to be clarified. World J Gastroenterol. 2019;25(22):2706-19.

25 Lin EW, Karakasheva TA, Hicks PD, Bass AJ, Rustgi AK. The tumor. microenvironment in esophageal cancer. Oncogene. 2016;35(41): 5337-49.

26 Rao CV, Reddy BS. NSAIDs and chemoprevention. Curr Cancer Drug Targets. 2004; 4: 29-42.

27 Alexandre L, Clark AB, Bhutta HY, Chan SS, Lewis MP, Hart AR. Association between statin use after diagnosis of esophageal cancer and survival: a Population-Based Cohort Study. Gastroenterology. 2016;150:854-7.

28 Agrawal S, Patel P, Agrawal A, Makhijani N, Markert R, Deidrich W. Metformin use and the risk of esophageal cancer in Barrett esophagus. South Med J. 2014;107:774-9. 